\title{
Sprachenstreit in Absurdistan
}

\section{Ulrich Nägeli}

Dr. med.

Bitte beachten Sie zu diesem Thema das Editorial von Christoph Hänggeli auf Seite 828 dieser Ausgabe.
Am 1.1. 2018 ist die Revision des Medizinalberufsgesetzes (MedBG) in Kraft getreten. Die FMH hat Ende 2017 darauf hingewiesen [1]. Nach Durchlesen dieses Artikels sah ich für mich als Arzt im Pensionierungsalter, der noch eine kleine Spezialsprechstunde in der Praxis meines Nachfolgers durchführte (verkehrsmedizinische Untersuchungen), keinerlei Handlungsbedarf. Ich habe zur Kenntnis genommen, dass es ein MedReg gebe, in welches ich nun automatisch eingetragen werde. Den zweiten Schwerpunkt, den Nachweis der Sprachkenntnisse, habe ich auch nicht auf mich bezogen. Ich komme nicht aus einem anderssprachigen Land und beherrsche drei unsere vier Landessprachen, zwei gut und eine leidlich. Wie sich die Revision des Gesetzes in andern Bereichen ausgewirkt hat, kann ich nicht beurteilen. Rasch gab es aber Probleme mit der Sprachregelung. Chr. Hänggeli hat in einem Editorial der SÄZ darauf hingewiesen: «Sprachkenntnisse ad Absurdum geführt. War die Politik 1877 weitsichtiger als 2018?» [2]. Ja, sie war es, das habe ich inzwischen selber feststellen müssen. Heute kann ich nur sagen, dass Weitsicht weiterhin fehlt und Einsicht nicht existiert,

\section{Riassunto}

L'iscrizione della competenza di lingua nel MedReg continua a causare problemi. Se certe istanze insistono su questa formalità, la situazione può diventare assurda. I criteri richiesti (certificato di lingua livello B2, lavoro di almeno tre anni in una regione della lingua in questione, non più vecchi di sei, rispettivamente 10 anni), oppure un genitore della lingua, non corrispondono alla realtà svizzera e sono troppo rigidi. Noi siamo abituati a comunicare regolarmente in altre lingue. Altri modi di acquisire la competenza linguistica sono esclusi, il MedReg non li accetta. Per i mediciTicinesi la certificazione della propria lingua è una pretesa assurda, ai giovani causa inoltre problemi se hanno studiato in una regione di una lingua e desiderano lavorare in una di un'altra. Un altro esempio è presentato: L'esperienza assurda di un medico in pensione al quale è stato chiesto da un collega di fare brevi supplenze nel suo studio (si conoscono da quando erano assistenti a Lugano). II DSS richiede l'iscrizione della conoscenza della lingua nel MedReg. II problema è che nessuno dei tre criteri previsti è soddisfatto. L'unico fatto è che parla I'Italiano, capisce i pazienti, anche se parlano in dialetto, e sa anche scriverlo in maniera accettabile.

jedenfalls nicht für Amtsschimmel, die hier viel Heu zum Verarbeiten gefunden haben. Hier ein Beispiel, das eine Glosse abgäbe, wenn es nicht eher zum Heulen wäre, und dann einige grundsätzliche Gedanken zum Thema. Aber der Reihe nach:

Vor gut einem Jahr hat mich ein Kollege im Tessin, wir kennen uns seit unserer Assistentenzeit in Lugano, gefragt, ob ich eine Stellvertretung bei ihm machen könnte. Nach einigem Zögern habe ich zugesagt und ihn im Sommer für 10 Tage in seiner Praxis abgelöst. Es ist uns dabei ein Formfehler bei der Meldung unterlaufen. Als er mich dann für einen weiteren kurzen Einsatz im Oktober angefragt hat, habe ich darauf bestanden, dass wir dies auch ordentlich melden. Ich habe es übernommen, diesen Brief zu verfassen und ihn dem Kantonsarzt geschickt. Das war offensichtlich die falsche Stelle. Erst nach der Stellvertretung habe ich Post bekommen, nicht vom Kantonsarzt, vom Departement (DSS). Es sei korrekt, wie von mir geschrieben, es brauche keine Bewilligung für eine kurze, nicht regelmässige Tätigkeit und unter 90 Tagen pro Jahr. Eine Meldung brauche es aber in jedem Fall. Bei einer künftigen Stellvertretung müsse ich dies rechtzeitig melden, und dann auch den Nachweis der Kenntnisse der Italienischen Sprache im MedReg eintragen lassen. Das hat mir nicht ganz eingeleuchtet. Gewohnt, Unklarheiten rasch zu beseitigen, habe ich zum Telefon gegriffen. Das Gespräch mit der zuständigen Person im Departement, in Italienisch, hat nichts gebracht. Es brauche fortan den Eintrag im Register.

Ein Anruf beim MedReg hat ergeben, dass es das wohl brauche; es gebe Kantone, die darauf bestünden. Das sei einfach, auch online zu erledigen, es koste Fr. 50.-. Was? Eine Gebühr für eine Ergänzung in einem Register?

Ich habe mich dann in diesem Medizinalpersonenregister gesucht, bin aber nicht fündig geworden. Die Rückfrage bei der Gesundheitsdirektion meines Wohnkantons hat ergeben, dass ich die Zulassung noch habe, es sei alles in Ordnung.

Nächster Anlauf beim MedReg: Eine kompetente Mitarbeiterin am Draht. Ihre erste Frage ist die nach dem Jahr des Staatsexamens. Ah, ja, das sei die Erklärung, die Jahrgänge mit Staatsexamen vor 1980 seien noch nicht erfasst. Ich würde ihr einen Gefallen tun, wenn ich meine Daten übermitteln könnte, die Suche im 
Archiv sei nämlich aufwändig. Grundsätzlich würden alle erfasst, welche beruflich aktiv seien. Diesen Gefallen konnte ich tun. Ich habe dann Auskunft erhalten, wie ich meine zusätzliche Sprache registrieren könne. Die Übernahme meiner Daten ins Register sei unentgeltlich, der Spracheintrag koste Fr. 50.-. Das ist mir dann plötzlich sauer aufgestossen; zufällig hatte ich gerade die Rechnung für die Direkte Bundessteuer erhalten; ich habe rasch hochgerechnet, dass ich gesamthaft, seit ich bundessteuerpflichtig bin, einen stattlichen sechsstelligen Betrag bezahlt habe; sicher für viele sinnvolle Verpflichtungen unseres Staates - und auch zur Erhaltung der Administration. Und nun sollte ich für einen läppischen Eintrag, ein Wort auf einer Zeile, noch 50 Franken nachreichen? Nächste Überraschung: Ich kann meine Sprachkenntnisse gar nicht eintragen lassen. Dazu müsste ich eines der folgenden Kriterien erfüllen: Diplom einer Sprachschule, Niveau B2 (nicht älter als sechs Jahre), Berufstätigkeit im Sprachgebiet während mindestens drei Jahren (nicht mehr als 10 Jahre zurückliegend) oder ein Elternteil mit dieser Sprache als Muttersprache. Ich habe nie einen solchen Sprachkurs besucht und damit auch kein Diplom. Die Grundlagen des Italienischen habe ich an der Mittelschule erhalten und die Sprache seither gebraucht. Ich habe mehrere
Jahre im Tessin gearbeitet, aber das liegt deutlich mehr als 10 Jahre zurück. Und dass ich 23 Jahre Militärdienst in den Tessiner Regimentern geleistet habe? Das gilt schon gar nicht. Mein Problem ist also, dass ich die Sprache verstehe, sogar einzelne Dialekte, sie spreche und auch schreiben kann, dies aber nicht anhand der doch recht willkürlicher Kriterien nachweisen kann. Was machen? Ja, ich könnte wohl an einer Sprachschule anfragen, ob ich eine solche B2-Prüfung ablegen könne, das ginge in meinem Fall vielleicht auch ohne einen Kurs zu absolvieren. Das würde mir nun aber

Ein Arzt müsste am Schluss seiner Aus- und Weiterbildung selbst einschätzen können, ob er einer Aufgabe gewachsen ist, fachlich und auch sprachlich.

nicht einmal im Traum einfallen! Aufgaben lösen wie «Ich habe meinen Zug um 15:04 verpasst, wann fährt der nächste Zug nach Basel?» ist wohl für den täglichen Bedarf nicht ganz unbedeutend, für unsern Beruf aber eher unerheblich. Da ist es wichtiger zu erfahren, ob es juckt, beisst, brennt oder schmerzt. Das lernt man bei der Arbeit und nicht in einer Sprachschule.

Ich habe dann vorgeschlagen, schriftlich Antrag auf Anerkennung meiner Sprachkompetenz zu stellen. Auf

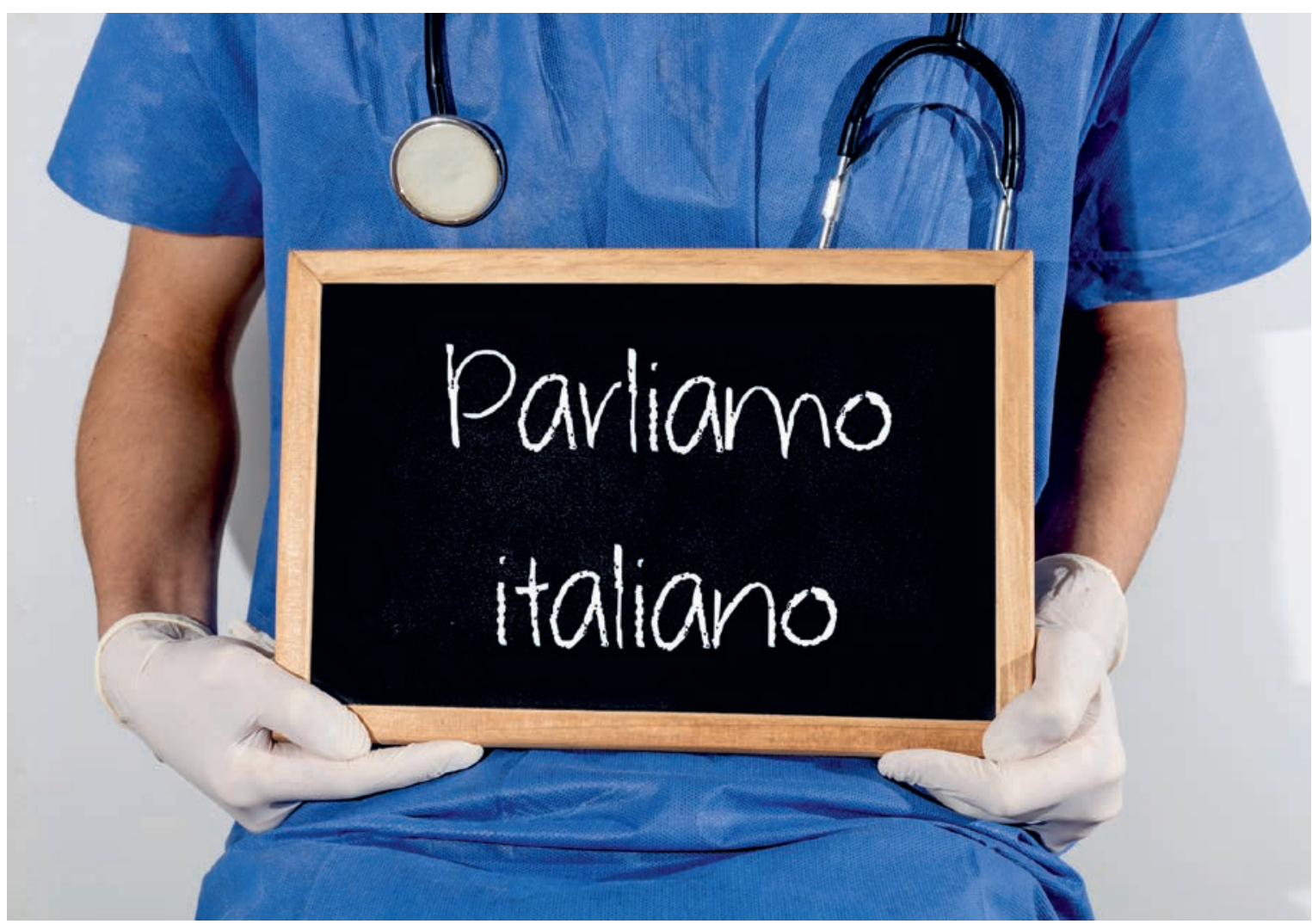

Für die für die jeweilige Berufsausübung notwendigen Sprachkenntnisse muss ein Nachweis erbracht und ein Eintrag im MedReg gemacht werden. 
Italienisch, selbst geschrieben und unkorrigiert. Darin habe ich Verständnis gezeigt, dass die Kriterien bei Ärzten aus Ländern mit einer einzigen Sprache oder einer andern Sprachkultur wohl eine Grundlage für die Aufnahme der Berufstätigkeit bei uns sein können. Für uns Schweizer seien sie aber ungeeignet. Wir lernen schon während des Studiums den Umgang mit anderssprachigen Patienten. Wir haben die Möglichkeit, einmal erlernte Landessprachen anzuwenden und aufzufrischen. Unser politisches System baut auf die Eigenverantwortlichkeit seiner Bürger. Ein Arzt (ich erlaube mir, weiter die männliche Form zu verwenden, da ich bis jetzt in der Ich-Form geschrieben habe, diese generellen Bemerkungen gelten natürlich auch für die Ärztin) muss am Schluss seiner Aus- und Weiterbildung selbst einschätzen können, ob er einer Aufgabe gewachsen ist, fachlich und auch sprachlich. Während der Weiterbildung oder bei einem Anstellungsverhältnis ist sein Arbeitgeber dafür zuständig, das gilt auch für die Sprachkompetenz. Das ist gesetzlich klar so geregelt. In meinem Antrag habe ich begründet, dass darum eine Selbstdeklaration der Sprachkompetenz genügen sollte. Schliesslich hat ja der Kollege, der mich für eine Stellvertretung anstellt, auch meine sprachliche Eignung beurteilt.

Der Antrag auf Eintrag der Sprachkenntnis ist abgelehnt worden. Die MEBEKO sei an die in der MedBV definierten Möglichkeiten für die Eintragung der Sprachkenntnisse ins MedReg gebunden. Eine Abweichung davon sei rechtlich unzulässig.

Es wird dabei ausdrücklich erwähnt, dass es jedem Arbeitgeber beziehungsweise der zuständigen kantonalen Behörde freistehe, anhand welcher Nachweise er/sie das Vorhandensein der für die jeweilige Berufsausübung notwendigen Sprachkenntnisse (Mindestniveau B2) überprüfen wolle.

Und so hat das zuständige Departement des Kantons Tessin dann, nach einigen weiteren Schlaufen, für das laufende Jahr 2019 die Bewilligung für Stellvertretungen erteilt. Für das nächste Jahr müsste das ganze Procedere wiederholt und dann auch der Nachweis der Sprachkompetenz erbracht und der Eintrag ins MedReg nachgeholt werden. Die Quadratur des Kreises soll also nochmals versucht werden.

Gerade während meiner zweiten Vertretung, im letzten Herbst, ist dann der OMCT, der Ordine dei Medici del Canton Ticino, aktiv geworden, weil die Tessiner
Kolleginnen und Kollegen die ganze Geschichte des Sprachnachweises ebenfalls als unzumutbar empfinden. Sie müssen die Kompetenz in ihrer eigenen Muttersprache nachweisen! Mehr als verständlich, dass sie das als Affront empfinden. Wer in der Romandie studiert hat, muss für eine Tätigkeit in der Deutschschweiz dann nochmals das ganze Procedere durchmachen. Sie haben Unterstützung durch Vorstösse zweier ihrer Parlamentarier in Bern erhalten, leider ohne Erfolg.

Die Sache hat einen sehr wichtigen anderen Aspekt. Man darf mit gutem Grund von einer staatspolitischen Dimension sprechen: Seit Jahren wird lamentiert, die Pflege der andern Landessprachen werde vernachlässigt. Es stimmt, unsere Landessprachen sind wegen der

Die natürlichste und einfachste Art, unsere Landessprachen zu pflegen und zu erhalten, wird durch administrative Hürden massiv erschwert.

Bedeutung des Englischen in vielen Bereichen, auch in der Medizin, unter Druck. Auf praktischer Ebene wird jetzt aber genau das Gegenteil getan: Unsere Landessprachen können wir in der Schule lernen. Die natürlichste und einfachste Art sie weiter zu pflegen und zu erhalten wird nun durch administrative Hürden massiv erschwert. Gerade für den Kanton Tessin besteht noch eine andere Gefahr: Eine Behinderung des Austauschs zwischen den Sprachregionen bedroht die Weiterbildung: Der bisher ganz normale Wechsel zwischen Sprachregionen wird beeinträchtigt, und solche Aktionen gefährden schliesslich auch den nationalen Zusammenhalt.

Ein weiterer Fakt ist, dass unsere Institutionen, ich denke hier z.B. das SIWF, auf unnötige Art zusätzlich belastet werden; diese Zeit und Arbeitskraft fehlt dann bei den Kernaufgaben.

\section{Bildnachweis}

(C) Francisco Solipa | Dreamstime.com

Literatur

1 Hänggeli C, Kuhn H. Das Medizinalberufsgesetz (MedBG) tritt am 1.1.2018 in Kraft. Schweiz Ärzteztg. 2017;98(51-52):1727-8.

2 Hänggeli C. Sprachkenntnisse ad Absurdum geführt. War die Politik 1877 weitsichtiger als 2018? Schweizerische Ärztezeitung 2018;99(16):497. 